\title{
An MRI compatible loading device for the reconstruction of clinically relevant plantar
} pressure distributions and loading scenarios of the forefoot

5

(1) CSHER, Faculty of Health Sciences, Staffordshire University, Stoke-on-Trent, United

9 Kingdom.

(*) Corresponding author, tel.: +44 1782295920

e-mail: panagiotis.chatzistergos@staffs.ac.uk

12

Keywords: Weight bearing MRI, Plantar pressure, tissue deformation, diabetic foot, joint stiffness, metatarsophalangeal joints, heel pad 


\section{Abstract:}

29 This study aims to present a new MRI compatible loading device capable of reconstructing realistic loading scenarios of the human foot for research in the field of foot biomechanics. This device has two different configurations: one used to compress the forefoot and one to bend the metatarsophalangeal joints. Required plantar pressure distribution under the metatarsal heads can be achieved by modifying the distribution of the dorsally applied forces. To validate the device, subjectspecific plantar pressures were measured and then reconstructed using the device. For quiet stance the peak pressure reconstruction error was $3 \%$ while for mid-stance phase of gait it was $8 \%$. The device was also used to measure the passive bending stiffness of the metatarsophalangeal joints of one subject with low intra-subject variability. A series of preliminary MRI scans confirmed that the loading device can be used to produce static weight-bearing images of the foot (voxel size: $0.23 \mathrm{~mm} \times 0.23 \mathrm{~mm} \times 1.00 \mathrm{~mm})$

The results indicate that the device presented here can accurately reconstruct subject specific plantar pressure distributions and measure the foot's metatarsophalangeal passive stiffness. Possible future applications include the validation of finite element models, the investigation of the relationship between plantar pressure and internal stresses/strains and the study of the foot's inter-segmental passive stiffness. 


\section{Introduction}

57 Investigating the internal stresses and strains of the human foot soft tissues is crucial to the understanding of foot biomechanics [1,2]. The significance of internal tissue stress and strain is even more pronounced in the case of the diabetic foot. Previous research indicates that ulceration begins internally and could be caused by deep tissue trauma [3]. In this context a number of different methodologies have been developed for the direct measurement of internal strains using medical imaging [2,4-12]. These protocols usually involve a comparison of an unloaded image of the tissue's structure against the images of the loaded structures under a range of externally applied loads. The implementation of this concept requires a method with three main constituents: a medical imaging modality, a tissue-loading device and an algorithm/procedure to quantify and map the changes of the tissues' structure [10].

67

From the common imaging modalities Magnetic Resonance Imaging (MRI) appears to be the most suited one for a detailed analysis of the three dimensional (3D) field of internal deformations of soft tissues [2]. MRI offers a superb contrast for the imaging of soft tissues and it doesn't employ any ionizing radiation. However, one of the main disadvantages of using MRI is the fact that applying loads in a controlled and repeatable manner inside an MRI scanner is considerably more difficult compared to other available modalities such us ultrasonography [4], fluoroscopy [5,6] or computer tomography (CT) [7-9]. The use of MRI imposes a number of limitations in terms of the dimensions and the materials that can be used to build a loading device, hence a number of studies have looked at tackling this challenge for various anatomical regions [2,13].

To investigate the internal deformations of the foot, Petre et al. [2] developed a sophisticated MRI compatible loading device capable of applying both normal and shear loads to the forefoot. The pilottesting of this device indicated that despite its capacity to apply "gait-like loading” [2] it couldn't generate plantar pressure distributions similar to the ones measured during walking. This was attributed to the fact that the loading device simultaneously applied a net force to both the metatarsal 
85 In contrast to internal deformations, the internal stresses of plantar soft tissues cannot be directly measured, but they can be estimated with the use of finite element (FE) models [1,10]. An accurate 3D FE model of the foot though, requires a large amount of information on its internal structure and the mechanical properties of its soft tissues (i.e. skin, fat, muscle etc.). Furthermore to accurately replicate realistic loading scenarios such models need information on the function and stiffness of its numerous joints and they have to be validated against experimental data.

91

The bending stiffness of joints in general can be analysed in two components: a dynamic one associated with active muscle forces and a passive one associated with the elastic properties of noncontractile tissues [14]. Hence measuring the passive stiffness of the joints of the foot in addition to enhancing our understanding on their function it can also give clinically relevant information about the mechanical status of non-contractile tissues, such as ligaments, tendons etc.

In this context the present study aims to present a novel MRI compatible device for the accurate reconstruction of subject specific plantar pressure distributions of the forefoot and the simulation of clinically relevant loading scenarios. This device was designed and built to enable the study of the invivo mechanical behaviour of the plantar soft tissues of the forefoot under compression as well as for the study the mechanical behaviour of the passive foot under bending.

\section{Participants and methods}

A total of six healthy volunteers (4 female, 2 male) with an average age of $33.0( \pm 6.3)$ years and average body mass of $70.9( \pm 7.7) \mathrm{kg}$ were recruited for this study (Table 1). Ethical approval was sought and granted by the University Ethics Committee and the volunteers provided full informed consent.

The MRI compatible loading device was designed using SolidWorks ${ }^{\circledR} 2010$ (Dassault Systèmes, Paris, France). The device consisted of custom made parts milled from polypropylene blocks and connected 
with Nylon plastic screws and bolts. The device (Figure 1) has a fixed part (A) for foot support and a movable one (B) for loading. The movable part (B) can rotate around a predefined axis (C). The device can be configured so that the initial angle between part B and the plantar surface of part A is either $90^{\circ}$ or $0^{\circ}$. Each one of these two configurations enables the application of different load scenarios (compression or bending modes respectively). In both cases the loading is applied with the help of a suspended MRI compatible mass. The suspended mass can be up to $5 \mathrm{Kg}$ which generates a maximum compressive force of $\approx 250 \mathrm{~N}$ or bending moment of $\approx 7 \mathrm{Nm}$. During the design of the loading device special attention was paid to minimize its dimensions and make sure it will fit inside a typical MRI scanner with a bore aperture of $60 \mathrm{~cm} \mathrm{[15].}$

\subsection{Compression mode}

The loading device is capable of applying known compressive forces at the dorsal side of the forefoot. For this purpose the movable part B (Figure 1A) is positioned at $90^{\circ}$ with respect to the foot-support (A) and equipped with a compression punch (D). This punch is used to compress the forefoot and to control the distribution of the applied load. More specifically a series of screws is used to modify the profile of the contact area between the punch and the dorsal surface of the foot (Figure 1A). The relative position of the foot and punch can also be modified with the use of $5 \mathrm{~mm}$ thick plastic sheets (E). Positioning the compression punch at the dorsal side of the MTHs enables the controlled loading of the soft tissues that lie directly below them. When a compressive load is applied at the dorsal side of the forefoot these non-contractile soft tissues (mainly skin and fat) are compressed between the foot-support (A) and the MTHs.

The ability of this device to reconstruct subject specific plantar pressure distributions was assessed in a pilot study. More specifically the loading device was used to reconstruct the plantar pressure developed at the MTH region during quiet stance and the mid-stance phase of walking gait.

The quality of the MRI images that can be recorded when the foot is under the static weight bearing conditions generated by the device was assessed in a series of preliminary scanning sequences. The 
forefoot region of a volunteer was compressed with a net force of $250 \mathrm{~N}$ and imaged using a $1.5 \mathrm{~T}$ MRI scanner. The duration of the scanning process was $\approx 4$ min and coronal T1 weighted 3D Fast Field Echo (FFE) images (Figure 2) were recorded with in-plane and out of plane resolution of 0.23 $\mathrm{mm}$ and $1.00 \mathrm{~mm}$ respectively. No plantar pressure measurement was performed inside the MRI scanner.

\subsubsection{Quiet stance}

In the case of quiet stance the average peak pressure under each Metatarsal Head (MTH) was measured for each of the six volunteers using a plantar pressure sensor (F-scan ${ }^{\circledR}$, Tekscan, Boston, MA, US) (Figure 1A). The volunteers stood on the sensor and the area under each MTH was identified by applying pressure manually at the dorsal side of the foot. The relative location of each MTH on the pressure sensor was utilized to measure the peak plantar pressure of each MTH (Figure 3). The plantar pressure was recorded at a sampling rate of $100 \mathrm{~Hz}$ for $10 \mathrm{sec}$ of quiet stance and the average peak pressure for each MTH was calculated to produce the reference measurements for the reconstruction. The relative position of the foot and the sensor was fixed using double sided tape. The volunteers' feet were then placed inside the loading device and a pressure was applied at the dorsal side of their forefoot. The suspended mass was gradually increased (in increments of $0.5 \mathrm{Kg}$ ) to approximate the total forefoot force. The profile of the punch was sequentially modified starting from the MTH with the maximum reference pressure and then moving medially and laterally to reconstruct the pattern of the reference plantar pressure distribution. This procedure was repeated until the difference between the reference and the reconstructed peak pressures was less than $5 \%$. After achieving a satisfactory replication of the overall pattern of pressure distribution, the plantar pressure was recorded again for 10 seconds at a sampling rate of $100 \mathrm{~Hz}$. One additional pressure measurement was performed for a single participant (participant \#6) with the compression punch completely flat (i.e. before any modification of its profile) to highlight the versatility of the loading device.

The duration of loading was decided based on preliminary results indicating that the plantar pressure distribution generated by the loading device stays practically constant for long periods of time. More 
specifically a net compressive force of $150 \mathrm{~N}$ was applied to the forefoot of a volunteer and the peak plantar pressure was recorded at $1 \mathrm{~Hz}$ for 20 minutes of continuous loading. The average value and standard deviation of the peak pressure was calculated.

The reproducibility of the results was assessed through a test re-test procedure (Table 1). After completing all tests, the loading procedure and pressure measurements were repeated for one volunteer (\#5) without reconfiguring the loading device (i.e. the suspended mass and the profile of the compression punch was the same for both test and re-test).

\subsubsection{Mid-stance phase of gait}

In the case of the mid-stance phase of gait the plantar pressure of a complete gait cycle was recorded for a single volunteer (\#5) using a walkway pressure mapping system (Walkway®, Tekscan, Boston, MA, US). An automated procedure within the Walkway® system was used to identify the area below each MTH and to measure the corresponding pressure in each region. In this case the reference values for the reconstruction procedure were the peak pressures under the MTHs for a randomly selected instance of the mid-stance phase of gait (50\% of mid-stance). The plantar pressure developed inside the loading device was measured again using F-scan®.

\section{$2.2 \quad$ Bending mode}

Reconfiguring the loading device allows the application of known bending moments to the foot. For this purpose parts $\mathrm{A}$ and $\mathrm{B}$ are positioned parallel to one another and the compression punch is removed (Figure 1B). In this configuration a rotation of part B around its axis $(\mathrm{C})$ tends to bend the subject's foot instead of compressing it. The relative position of the foot with respect to the rotation axis can be modified with the use of $5 \mathrm{~mm}$ thick plastic sheets (E) (Figure 1B). These sheets are placed between the foot and the foot's support elevating the foot along two axes: one parallel and one perpendicular to the plantar surface (Figure 1B). When the device is used inside an MRI scanner the relative angle between parts A and B can be measured from the MRI images. 
The bending mode of the MRI compatible loading device was pilot tested for a single volunteer by measuring the bending stiffness of the metatarsophalangeal (MP) joints. For this purpose the bending axis of the volunteer's MP joints was first identified based on anatomical landmarks [16]. The volunteer's foot was fixed inside the device using Velcro straps (Figure 1B) and using a goniometer the bending angle of the MP joints was measured for bending moments ranging from $1.8 \mathrm{Nm}$ to 4.5 Nm. Ten preconditioning load/unload cycles were performed before each measurement and each measurement was repeated five times.

\section{Results}

\subsection{Compression mode}

The pilot MRI scan indicated that the quality of the MRI images recorded when the foot is under the static weight bearing conditions generated by the device have a resolution that is high enough to enable the identification and segmentation of different tissues of the foot (Figure 2).

\subsubsection{Quiet stance}

In the case of quiet stance the average difference between the reference and the reconstructed total maximum pressures was only $4 \%$ while the mean difference for all MTHs ranged between $2 \%$ and 15\% (Table 1). In all cases the total maximum pressure was observed under the same MTH for both the reconstructed and the reference pressure distributions. As it can be seen in Table 1 and Figure 3 the loading device was capable of reconstructing different and distinctive subject specific loading patterns. The ability of the device to modify the distribution of plantar pressures becomes clearer if one compares the initial pressures that are measured in the device before any adjustment of the compression profile (i.e. for a compression profile that is completely flat) to the final ones after all necessary adjustments had been completed (Figure 3).

Moreover the loading device was capable of maintaining a constant distribution of plantar pressure for long periods of time. The average peak pressure measured for 20 minutes of continuous loading was equal to $111.8 \mathrm{kPa}$ with a standard deviation of only $4.3 \mathrm{kPa}$ (i.e. $3.8 \%$ error). In terms of 
reproducibility, the test-retest procedure yielded differences in terms of average peak pressures that were lower than $5 \%$ (Table 1).

\subsubsection{Mid-stance phase of gait}

In the case of mid-stance phase of gait the average difference between the reference peak pressures and the reconstructed average peak pressures was $8 \%$ (Table 1). The difference between the overall maximum values was also $8 \%$.

\subsection{Bending mode}

The standard deviation of the measured rotation angles for different values of the externally applied moment was always lower than 3 deg indicating a relatively low intra-subject variability (Table 2). Moreover, bending angle appeared to increase linearly with moment $\left(\mathrm{R}^{2}=0.98\right)$. The slope of the moment/angle graph was equal to $0.06 \mathrm{Nm} / \mathrm{deg}$.

\section{Discussion}

The purpose of this study was to present a new foot loading device that is capable of reconstructing clinically relevant loading scenarios inside an MRI scanner. This device was initially designed to enable the validation of 3D FE models of the foot and more specifically to help assess their accuracy to predict the internal stresses and strains of plantar soft tissues and to simulate the function of the foot's joints. For this reason a custom made device was designed to allow two different configurations for the application of different loads, i.e. compression and bending. Having two different configurations for compression and bending enabled the realisation of different loading scenarios using a single device instead of two while at the same time helped simplifying the design of the device.

The new method for the reconstruction of subject specific pressure distributions presented here was focused on the MTH area. The MTH area along with the heel and the hallux are the areas with the highest ulceration rates in people with diabetes. A recent study by Ledoux et al. [17] demonstrated for 
the first time that the ulceration risk for the MTH area is correlated to in-shoe peak plantar pressure. No similar correlation was found for the heel and hallux which could indicate that in the case of the MTH area ulceration is caused by normal loading instead of shear while in the case of the heel and hallux shear loading (among other reasons) could be the main risk factor. These results highlight the importance of studying the in-vivo mechanical behaviour of the plantar soft tissues of the MTH area and the clinical relevance of analysing their internal strain fields with the help of medical imaging.

There are two ways to load the forefoot inside a typical MRI scanner: either by supporting the dorsal side of the passive foot and loading the plantar one [2] or by supporting its plantar side and loading its dorsal one. Considering that pressure is developed at both sides of the foot regardless of the direction of the externally applied load, it becomes clear that the deformations of the dorsal soft tissues will always be unrealistic. Indeed these tissues are not normally meant to be subjected to large compressive loads. On the other hand the plantar soft tissues of the MTH area are compressed between the plantar surface of the loading device and the MTHs. This compressive load within the internal structures will be generated regardless of the direction of the external load either from the dorsal or the plantar aspect of foot. In addition, this scenario closely simulates the loading conditions of quiet stance and the mid-stance phase of gait. The only way to validate the accuracy of this reconstructed internal loading condition is with the help of upright weight bearing MRI, which is beyond the scope of this manuscript.

In a previous attempt to simulate clinically relevant loading scenarios inside an MRI scanner Petre et al. [2] developed a loading device that was capable of applying "gait-like loading" [2] but couldn't reconstruct subject specific plantar pressure distributions. According to the results presented by Petre et al. [2], the average difference in terms of peak pressure between the reference and the reconstructed loading was $66 \%$ while only in $50 \%$ of the cases the reconstructed peak pressure was observed under the correct MTH (i.e. the same MTH as in the reference pressure distributions) [2]. This was attributed to the lack of control over the distribution of the load and to the fact that the device applied a net force simultaneously to both the MTH and toe regions [2]. 
281 In order to address this issue the device presented here was equipped with a specially designed punch.

282 This punch is used to compress the forefoot and modify the distribution of the applied load. This unique feature allowed the accurate reconstruction of diverse and distinctive subject specific plantar pressure distribution patterns. Indeed the average difference between the reference and the reconstructed maximum pressures was only $4 \%$ and in every case the maximum pressure was observed under the same MTH for both the reconstructed and the reference pressure distributions. Based on these results it can be concluded that the device presented here offers a clear improvement for the reconstruction of subject specific plantar pressure distributions of the MTH area.

The reproducibility of loading with an accuracy of $5 \%$ that was assessed through a test-retest procedure allows the reconstruction of subject specific loading conditions inside an MRI scanner. This allows configuring the device before entering the MRI environment with the use of pressure measurement sensors that are not usually MRI compatible and then to reproduce the same loading conditions inside the scanner.

Another challenge stems from the fact that MRI scanning can be a rather lengthy process and therefore loading should be kept constant for a significant amount of time. The device presented here was proven capable of keeping loading constant for up to 20 minutes (net force $=150 \mathrm{~N}$, average peak pressure $=111.8 \mathrm{kPa}$ ). Even though the results indicated that even higher loads could be sustained for even longer periods of time the actual limits both in terms of loading and time is more likely to be set based on considerations about the comfort and wellbeing of the subject rather than the capabilities of the device. With respect to this, special attention needs to be given to patients with impaired circulation and nerve damage to prevent trauma. Considering the limited access to the device inside the MRI scanner special care should be given to ensure that the toes of the subject are kept in a neutral position and that the skin at the dorsal side of the foot is not over-stretched as a result of loading. 
The second configuration of the device allows the application of bending moments. Even though this loading scenario does not simulate an everyday activity it can give clinically relevant information on foot biomechanics. Imaging the foot under bending loads can shed light on the function of the joints of the foot and the stiffness of their non-contractile tissues, like ligament and tendon stiffness, with implications in assessing the functional impairment in conditions like diabetes.

In the context of this study the bending stiffness of the MP joints of one subject with no known musculoskeletal abnormality or diabetes was measured for validation and demonstration purposes. As expected, the passive bending stiffness of the MP joints measured here was significantly lower compared to measurements taken during locomotion. Oleson et al.[16] studied the stiffness of the forefoot during running with the use of a motion analysis system. They concluded that the MP joints have a time-depended stiffness and its function resembles that of an "active mechanism" instead of a linear spring [16]. The bending stiffness reported by Oleson et al. [16] was close to $1.6 \mathrm{Nm} / \mathrm{deg}$ for a flexion angle of 20 deg while the respective value of the passive stiffness measured here was only $0.06 \mathrm{Nm} / \mathrm{deg}$.

No specific conclusions can be drawn on the mechanical behavior of the MP joints at this point due to the nature and design of this pilot investigation. Although the bending tests were performed on a single subject, further studies will use this device to measure the passive MP joint stiffness of people with no musculoskeletal abnormalities or diabetes as well as of people with diabetes to study differences in terms of joint mobility and stiffness. Previous investigations indicate that people with diabetes are often found to have limited joint mobility which is linked to increased ulceration risk [19]. Increased joint stiffness caused by non-enzymatic glycosylation is presumed to be one of the main contributors of limited joint mobility of the diabetic foot's distal joints [19]. Studying the passive bending stiffness and the mobility of the joints within the diabetic foot will shed light on the causative factors of this pathological change with the potential to help improve current therapeutic approaches for the reduction of ulceration rates. 
336 The type and range of loading scenarios that can be realised using this device is mainly limited by the

337 fact that in its current form it cannot apply combinations of different types of loading such as compression and bending or combined compression and shear. For this reason the plantar pressure reconstructions performed here were limited to load cases where shear loads are minimal such as quiet stance and the mid-stance phase of gait. Despite this limitation the device could be used with minor modifications to study loading scenarios involving compression of the forefoot at different dorsiflexion angles of the toes. The dorsiflexion angle of the toes could be modified easily with the use of wedges with different inclinations. Another limitation of this device is that it cannot be used to study rate dependent phenomena.

For the purposes of the present study the maximum compressive force applied to a subject was $250 \mathrm{~N}$. Even though this force may seem relatively low compared to the net forces applied to the foot during everyday activities the results of this study indicate that if this force is properly focused it can generate plantar pressures in order of magnitude that are relevant to the cases of quiet stance and midstance phase of walking gait. The maximum net compressive force that can be applied by the device is mainly limited by the fact that pressure is applied on a relative small area at the dorsal side of the foot. Because of that increased loads could cause discomfort or trauma. Even though the simulation of loading scenarios involving higher compressive forces at the MTH area (i.e. similar or higher that body weight) were beyond the scope of this study, minor modifications could increase the magnitude of the compressive force that can be safely applied. Increasing the contact area between the compression punch and the dorsal side of the foot would allow the application of significantly higher forces.

The MRI compatible loading device presented here can realise clinically relevant loading scenarios and allows the accurate reconstruction of subject specific plantar pressure distributions of the MTH area for the cases of quiet stance and mid-stance phase of gait. The device can be used to validate numerical models of the foot, where the loading is applied by a virtual punch. The validated foot models can then be used to simulate real-life scenarios. In the future this device can be used for the 
364 as for the study of the forefoot's inter-segmental passive stiffness.

365

\section{Acknowledgments}

367 Funding from DiabSmart project is acknowledged. DiabSmart project was funded by the European (FP7-PEOPLE-2011-IAPP). This project has a focus on development of a new generation of Diabetic footwear using an integrated approach and Smart materials.

Competing interests: None declared 
[1] Yarnitzky G, Yizhar Z, Gefen A. Real-time subject-specific monitoring of internal deformations and stresses in the soft tissues of the foot: A new approach in gait analysis. J Biomech 2006;39:2673-89.

[2] Petre MT, Erdemir A, Cavanagh PR. An MRI-compatible foot-loading device for assessment of internal tissue deformation. J Biomech 2008;41:470-4.

[3] Gefen A. Plantar soft tissue loading under the medial metatarsals in the standing diabetic foot. Med Eng Phys 2003;25:491-9.

[4] Hsu C-C, Tsai W-C, Hsiao T-Y, Tseng F-Y, Shau Y-W, Wang C-L, et al. Diabetic effects on microchambers and macrochambers tissue properties in human heel pads. Clin Biomech (Bristol, Avon) 2009;24:682-6.

[5] Gefen A, Megido-Ravid M, Itzchak Y, Arcan M. Biomechanical analysis of the threedimensional foot structure during gait: a basic tool for clinical applications. J Biomech Eng 2000;122:630-9.

[6] Gefen A, Megido-Ravid M, Itzchak Y. In vivo biomechanical behavior of the human heel pad during the stance phase of gait. J Biomech 2001;34:1661-5.

[7] Smith KE, Commean PK, Mueller MJ, Robertson DD, Pilgram T, Johnson J. Assessment of the diabetic foot using spiral computed tomography imaging and plantar pressure measurements: a technical report. J Rehabil Res Dev 2000;37:31-40.

[8] Madsen MT, Haller J, Commean PK, Vannier MW. A device for applying static loads to prosthetic limbs of transtibial amputees during spiral CT examination. J Rehabil Res Dev 2000;37:383-7.

[9] Commean PK, Mueller MJ, Smith KE, Hastings M, Klaesner J, Pilgram T, et al. Reliability and validity of combined imaging and pressures assessment methods for diabetic feet. Arch Phys Med Rehabil 2002;83:497-505.

[10] Petre MT, Erdemir A, Panoskaltsis VP, Spirka TA, Cavanagh PR. Optimization of nonlinear hyperelastic coefficients for foot tissues using a magnetic resonance imaging deformation experiment. J Biomech Eng 2013;135:61001-12.

[11] Gefen A. Integration of plantar soft tissue stiffness measurements in routine MRI of the diabetic foot. Clin Biomech 2001;16:921-5.

[12] Weaver JB, Doyley M, Cheung Y, Kennedy F, Madsen EL, Van Houten EEW, et al. Imaging the shear modulus of the heel fat pads. Clin Biomech (Bristol, Avon) 2005;20:312-9.

[13] Elbannan KM, Salisbury SP. Design of a two degree-of-freedom, MRI-compatible actuator. Conf Proc IEEE Eng Med Biol Soc 2012;2012:940-3.

[14] Roy A, Forrester LW, Macko RF, Krebs HI. Passive and active wrist joint stiffness following eccentric exercise. J Rehabil Res Dev 2013;50:555-72. 
[15] Uppot RN, Sahani D V, Hahn PF, Gervais D, Mueller PR. Impact of obesity on medical imaging and image-guided intervention. AJR Am J Roentgenol 2007;188:433-40.

[16] Oleson M, Adler D, Goldsmith P. A comparison of forefoot stiffness in running and running shoe bending stiffness. J Biomech 2005;38:1886-94.

[17] Ledoux WR, Shofer JB, Cowley MS, Ahroni JH, Cohen V, Boyko EJ. Diabetic foot ulcer incidence in relation to plantar pressure magnitude and measurement location. J Diabetes Complications 2013;27:621-6.

[18] Cavanagh PR, Ulbrecht JS. The biomechancis of the foot in diabetes mellitus. In: Bowker JH, Pfeifer MA, editors. Levin O’Neal's Diabet. Foot, Philadelphia: Elsevier Health Sciences; 2008, p. 115-84. 
Table 1: The reference and the reconstructed average peak pressures measured under each MTH for the case of quiet stance (six volunteers) and the respective reference peak pressures and reconstructed average peak pressures (one volunteer) for the case of mid-stance phase of gait. The overall maximum values for each volunteer are highlighted.

\begin{tabular}{|c|c|c|c|c|c|c|c|c|c|c|}
\hline & & & \multirow[b]{2}{*}{ \#1 } & \multicolumn{6}{|c|}{ Quiet stance } & \multirow{2}{*}{$\begin{array}{c}\begin{array}{c}\text { Mid- } \\
\text { stance }\end{array} \\
\# 5\end{array}$} \\
\hline \multicolumn{2}{|c|}{ Volunteer: } & & & $\# 2$ & \#3 & $\# 4$ & \#5 & $\# 5$ & \#6 & \\
\hline \multicolumn{3}{|r|}{ Gender (M/F): } & $\mathbf{F}$ & $\mathrm{M}$ & $\mathbf{F}$ & T & & & $\mathbf{E}$ & $\mathbf{F}$ \\
\hline \multicolumn{3}{|r|}{ Age (y) } & 32 & 35 & 15 & 38 & 30 & 30 & 24 & 30 \\
\hline \multicolumn{3}{|r|}{ Weight (Kg): } & 60 & 82 & 72 & 82 & 67 & 67 & 70 & 67 \\
\hline \multirow{10}{*}{ 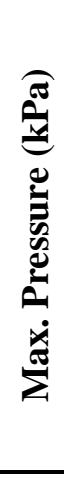 } & 1st & Reference/ & $29 /$ & $176 /$ & $46 /$ & 691 & $218 /$ & $218 /$ & $50 /$ & $57 /$ \\
\hline & MTH & Reconstructed & 26 & 163 & 46 & 70 & 221 & 211 & 58 & 65 \\
\hline & 2nd & Reference/ & $70 /$ & $60 /$ & $79 /$ & 641 & $66 /$ & $66 /$ & 511 & 971 \\
\hline & MTH & Reconstructed & 78 & 67 & 82 & 64 & 64 & 70 & 69 & 97 \\
\hline & 3rd & Reference/ & $51 /$ & 881 & $95 /$ & $60 /$ & $77 /$ & $77 /$ & $77 /$ & 981 \\
\hline & MTH & Reconstructed & 52 & 90 & 91 & 57 & 42 & 46 & 76 & 106 \\
\hline & 4th & Reference/ & $53 /$ & $167 /$ & $57 /$ & 491 & $49 /$ & $49 /$ & 411 & $91 /$ \\
\hline & МТH & Reconstructed & 53 & 151 & 80 & 50 & 40 & 42 & 35 & 85 \\
\hline & 5th & Reference/ & 421 & 1841 & 611 & 681 & $37 /$ & $37 /$ & $37 /$ & 921 \\
\hline & МТН & Reconstructed & 41 & 185 & 62 & 66 & 35 & 38 & 38 & 81 \\
\hline & & Iean error $(\%)$ : & 5 & 6 & 10 & 2 & 15 & 15 & 14 & 8 \\
\hline
\end{tabular}

Table 2: The results from the pilot testing of the device's bending mode. The bending angle values of all five trials are presented.

\begin{tabular}{c|ccccc|c|c}
$\begin{array}{c}\text { Bending } \\
\text { moment }\end{array}$ & Trial & Trial & Trial & Trial & Trial & Average & STDEV \\
$\mathbf{( N m )}$ & 1 & 2 & 3 & 4 & 5 & & \\
\hline $\mathbf{1 . 8}$ & 89 & 90 & 90 & 90 & 90 & $\mathbf{9 0}$ & $\mathbf{0}$ \\
\hline $\mathbf{2 . 2}$ & 105 & 99 & 103 & 103 & 103 & $\mathbf{1 0 3}$ & $\mathbf{2}$ \\
\hline $\mathbf{3 . 3}$ & 120 & 120 & 119 & 119 & 124 & $\mathbf{1 2 0}$ & $\mathbf{2}$ \\
\hline $\mathbf{3 . 9}$ & 126 & 123 & 127 & 127 & 127 & $\mathbf{1 2 6}$ & $\mathbf{2}$ \\
\hline $\mathbf{4 . 6}$ & 133 & 138 & 139 & 140 & 138 & $\mathbf{1 3 8}$ & $\mathbf{3}$
\end{tabular}


471 Figure 1: Two different configurations of the MRI compatible loading device used to apply

472 compressive (A) and bending loads (B). The punch used to apply the compressive load and to control 473 its distribution is also shown.

474

475 Figure 2: MRI images of the forefoot before (A) and after compression (B). The boundaries of the 476 foot and of the visible bones have been manually outlined for both cases (dotted blue and continuous 477 red curves for A and B respectively) and presented together (C) for comparison.

Figure 3: The reference planar pressures (left) and the ones measured inside the device before (centre) and after (right) the adjustment of the compression punch profile for volunteer \#6 (pressure in $\mathrm{kPa}$ ). The area of the MTHs is defined using a number of polygons. Inside each one of these polygons the location of peak pressure is marked by a rectangle. The average values of the peak pressures of each MTH are also plotted together for comparison. 
497 Figure 1:

498

(A)

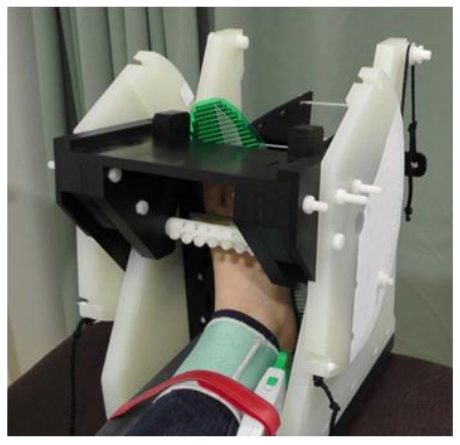

(B)
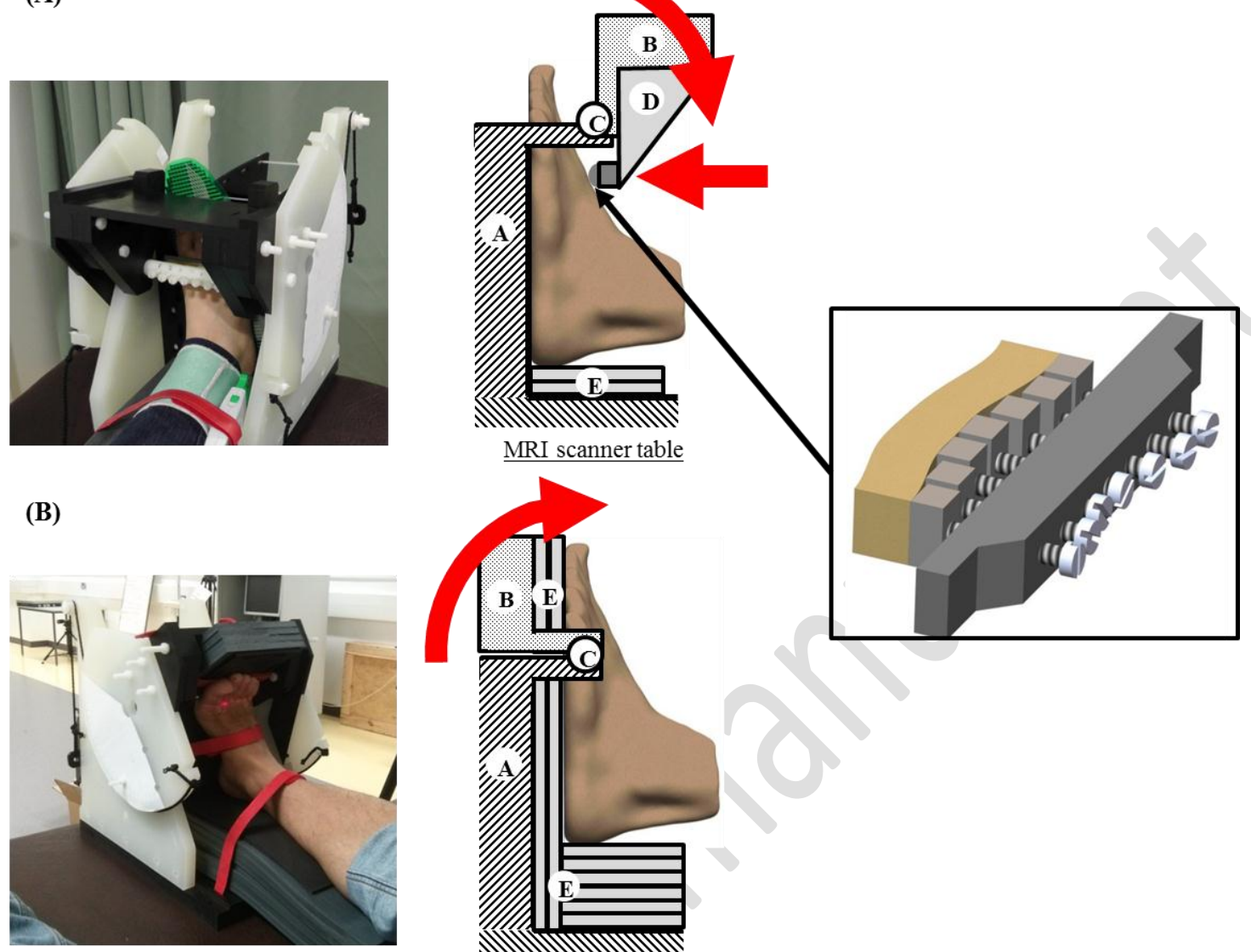

499

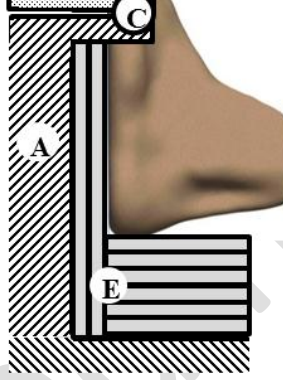

MRI scanner table

500

501

502

503

504

505

506

507

508

509

510 
511 Figure 2:

512

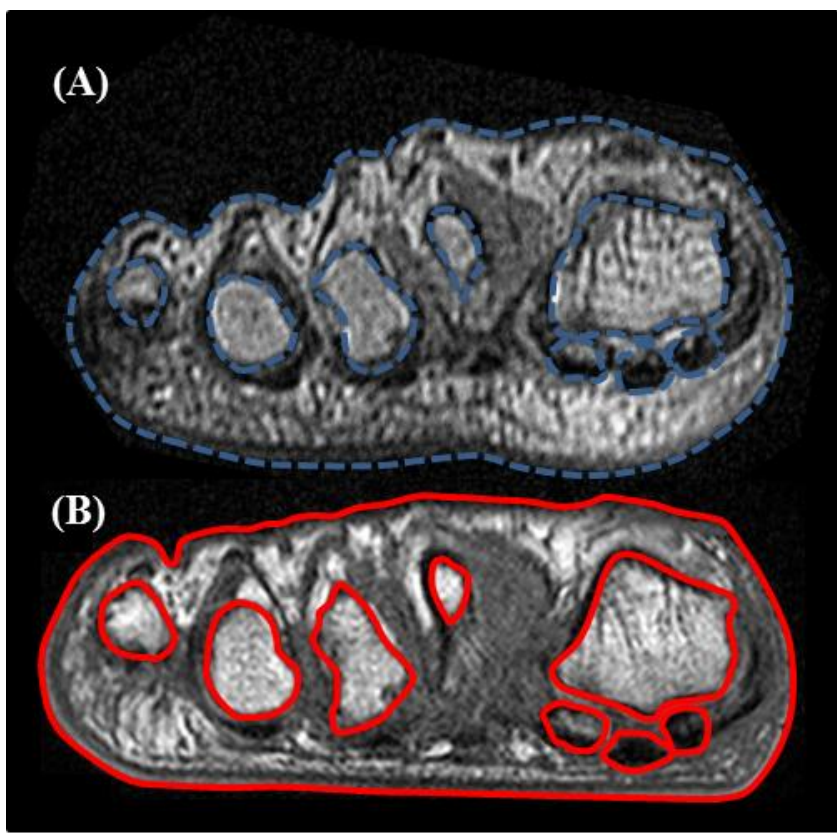

(C)

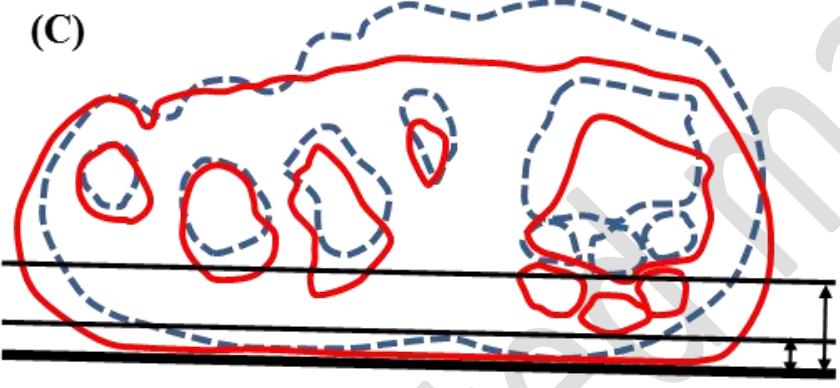

513

514

515

516

517

518

519

520

521

522

523

524 

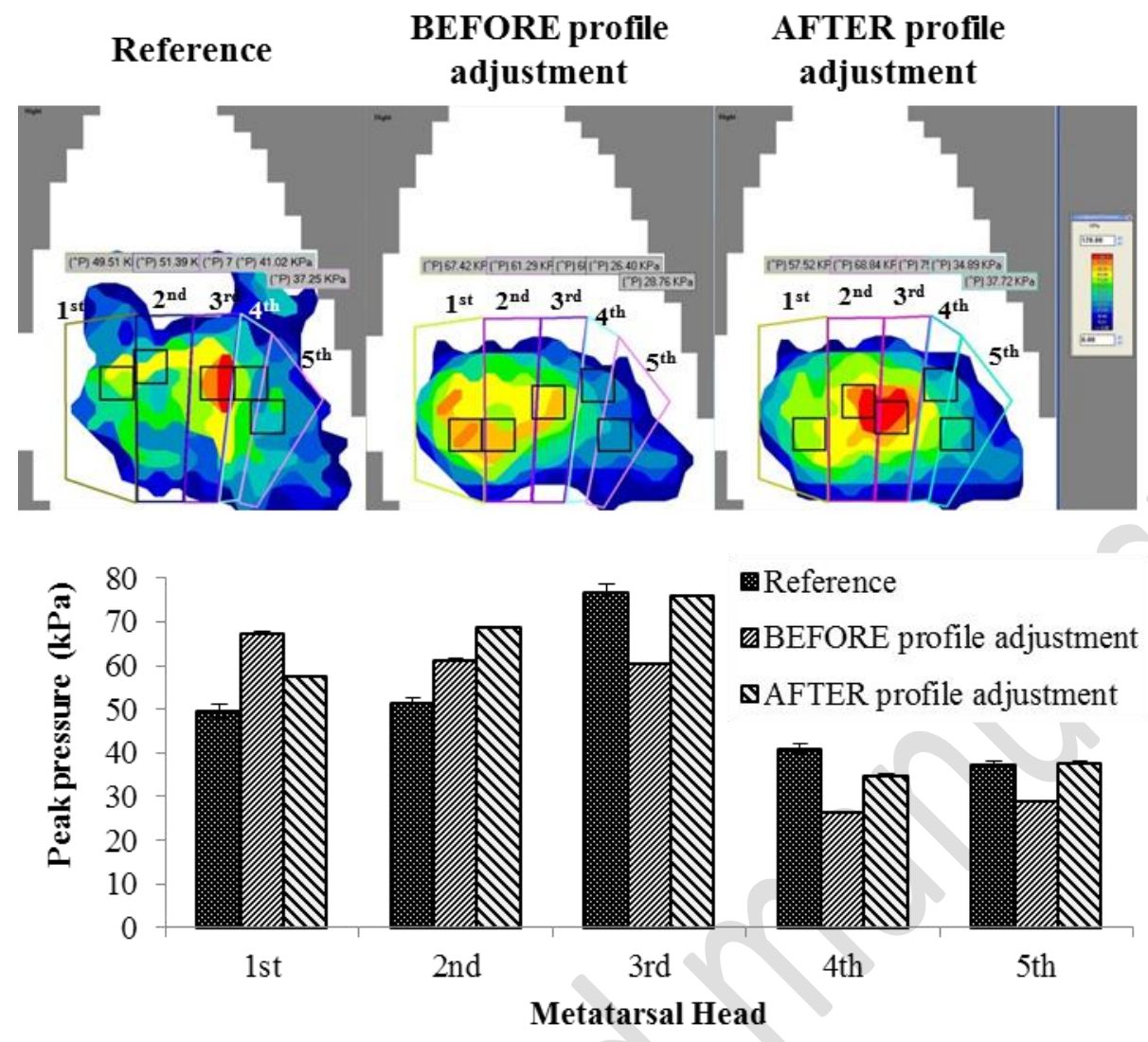

527

528

529

530

531

532

533

534

535

536

537 